\title{
A Biometric Solution for Door Locking System using Real time Embedded System and Arduino as the Microcontroller
}

\author{
Subhankar Chattoraj ${ }^{1}$, Karan Vishwakarma ${ }^{2}$ \\ ${ }^{I}$ Department of Electronics \& Communication Engineering Techno India University, Salt Lake Main Campus, \\ EM /4 Salt Lake \\ ${ }^{2}$ Department of Bio Technology Engineering Techno India University, Salt Lake Main Campus, EM /4 Salt Lake
}

\begin{abstract}
In today's world be it offices, laboratories, school or home the first thing which concern is the security. In order to make your data, money, premises and personal belonging safe and secured from unauthorized person. This paper represents a finger print recognition biometrics system based on real time embedded system which will provides a complete security solution and making unable to access for the unauthorized people. In comparison to the other methods authentication through RFID, passwords security this method has proven to be most efficient and reliable. Unauthorized access will be strictly prohibited by designing this system. The system stores the finger print of authorized people and only giving access to them. Fingerprint recognition is done by a sensor which can be connected with Arduino to validate for authentication. If the user's fingerprint has a positive match the door will open otherwise the GSM module gets triggered and the registered user gets a message and the buzzer connected will be initiated to alert the people or the security official in the surroundings.
\end{abstract}

Index Terms:R305, Arduino, Buzzer, GSM Modem, Security

\section{Introduction}

Security has become the primary concerned of most individual in this new era. To overcome it manually has become a real challenge for everyone. Instead, we found an alternative way which can help us to overcome our needs as well as automatized. In this automated world where technology is transforming very fast one can have access to information, database sitting at any parts of the world but it also comes with some great disadvantages. Pin verification, password protection, one time password (opt), barcode ID card and other identification system used can be copied or stolen by the imposters. One of the latest technologies is the finger print biometrics system which is now considered as one of the most efficient and trusted security system. The main reason for its reliability is that a finger print cannot have a positive match with someone else who is an unauthorized user. Each and every individual has a unique finger print and making it impossible to hack it. Many other biometrics technology such as retina, iris, voice recognition are also been a key subject of research and implementation nowadays but among these available biometric technology fingerprint is considered as having one of the least mismatch ratio and one of the most reliable in the security and surveillance world.

\section{Related Works}

Various attempts and research were made to overcome the security issue and still it undergoes.

\section{Lock and Key System}

First step towards security was Lock and key system. Security protocol followed in this system was "Single key for a single lock". Initially, this system was considered to provide utmost security but soon it proved to me wrong by the fact that multiple keys of a single lock can easily made. Hence this system is an outdated system to provide security.

\section{Password Authentication}

In the second level of security password [1-2] was used as an authenticating tool. In this type of system a multiple set of numbers or alphabets is stored in the database for the purpose of authentication. Only theauthorized user get to know the stored combination in the database so entering the correct combination results a positive match for the user. This system also have a disadvantage that password can be hacked or acquired by unauthorized user by continuously trying all the possible combinations.

\section{RFID Authentication}

Later a new technology for providing security was developed [3] "The authentication by RFID card". This system enriched the level of security. Access is granted only for the user whose RFID code has a positive match for authorized code. This system also have disadvantage of duplication of RFID card or by RFID skimming [4] and anyone who possess this card could unlock the door. 


\section{Hardware Platform}

The hardware part mainly consists of a digital computer, an Arduino Uno board, R305, 16x2 LCD displays, RS232 and a Buzzer which is being discussed along with their specific functions.

\section{Arduino Uno}

Arduino Uno [5-6] is a microcontroller board based on the ATmega328P. It has 14 digital input/output pins (of which 6 can be used as PWM outputs), 6 analog inputs, a 16 MHz quartz crystal, a USB connection, a power jack, an ICSP header and a reset button. Most boards include a $5 \mathrm{~V}$ linear regulator and a $16 \mathrm{MHz}$ crystal oscillator (or ceramic resonator in some variants), although some designs such as the Lily Pad run at $8 \mathrm{MHz}$ and dispense with the onboard voltage regulator due to specific form-factor restrictions. An Arduino's microcontroller is also pre-programmed with a boot loader that simplifies uploading of programs to the on-chip flash memory, compared with other devices that typically need an external programmer. This board has a 5 volt linear regulator and a $16 \mathrm{MHz}$ crystal oscillator.

\section{Finger Print Scanner Module (R305)}

R305 is a finger print sensor module [7] with TTL UART interface for direct connections to microcontroller UART or to PC through MAX232 / USB-Serial adapter. The user has compatibility to store the finger print data in the module and can configure it in 1:1 or 1: $\mathrm{N}$ mode for identifying the person. The FP module can directly interface with $3 \mathrm{v} 3$ or $5 \mathrm{v}$ Microcontroller. A level converter (like MAX232) is required for interfacing with PC serial port.Integrated image collecting and algorithm chip are together. Fingerprint reader can conduct secondary development and can be embedded into a variety of end products.R305 has Low power consumption, low cost, small size, and excellent performance professional optical technology. It also has good image processing capabilities i.e. can successfully capture image up to resolution $500 \mathrm{dpi}$.

\section{Liquid Crystal Display (LCD)}

Liquid Crystal Display screen [8-9] is an electronic display module and find a wide range of applications. A 16x2 LCD display is very basic module and is very commonly used in various devices and circuits. LCD stands for liquid crystal display. They come in many sizes $8 \times 1,8 \times 2,10 \times 2,16 \times 1,16 \times 2,16 \times 4$, $20 \times 2,20 \times 4,24 \times 2,30 \times 2,32 \times 2,40 \times 2$ etc. .These modules are preferred over seven segments and other multi segment LEDs. The reasons being: LCDs are economical; easily programmable; have no limitation of displaying special \& even custom characters (unlike in seven segments), animations and so on. A 16x2 LCD means it can display 16 characters per line and there are 2 such lines. In this LCD each character is displayed in 5x7 pixel matrix. This LCD has two registers, namely, Command and Data. The command register stores the command instructions given to the LCD. A command is an instruction given to LCD to do a predefined task like initializing it, clearing its screen, setting the cursor position, controlling display etc. The data register stores the data to be displayed on the LCD. The data is the ASCII value of the character to be displayed on the LCD.

\section{GSM Modem}

GSM/GPRS RS232 [10-11] Modem from rhydo LABZ is built with SIMCOM Make SIM900 Quadband GSM/GPRS engine, works on frequencies $850 \mathrm{MHz}, 900 \mathrm{MHz}, 1800 \mathrm{MHz}$ and $1900 \mathrm{MHz}$ It is very compact in size and easy to use as plug in GSM Modem. The Modem is designed with RS232 Level converter circuitry, which allows you to directly interface PC Serial port. The baud rate can be configurable from 9600115200 through AT command. Initially Modem is in Auto baud mode. This GSM/GPRS RS232Modem is having internal TCP/IP stack to enable you to connect with internet via GPRS. It is suitable for SMS as well as DATA transfer application in M2M interface GSM/GPRS MODEM RS232 is built with dual band GSM/GPRS engine SIM900A works on frequencies 900/ $1800 \mathrm{MHZ}$. The modem is coming with RS232 interface, which allows you connect PC as well as microcontroller with RS232 chip (Max232).The Baud rate is configurable from 9600-115200 through AT command. The GSM /GPRS modem is having internal TCP/IP stack to enable you to connect with interest via GPRS. It is suitable for SMS. Voice as well as DATA transfer application in M2M interface .The onboard regulated power supply allows you to connect wide range unregulated power supply. Using this modem, you can make audio calls, SMS, read SMS, attend the incoming calls etc. through simple AT commands.

\section{Servo Motor}

A servo motor [12] is closed-loop servomechanism that uses position feedback to control its motion and final position. The input to its control is some signal, either analogue or digital, representing the position commanded for the output shaft. The motor is paired with some type of encoder to provide position and speed feedback. In the simplest case, only the position is measured. The measured position of the output is compared to the command position, the external input to the controller.If the output position differs from that required, an 
error signal is generated which then causes the motor to rotate in either direction, as needed to bring the output shaft to the appropriate position. As the positions approach, the error signal reduces to zero andthe motor stops automatically.

\section{Methodology}

In this paper our main aim is to propose model for finger print biometric security. Proposed system architecture is shown in Figure 1.1

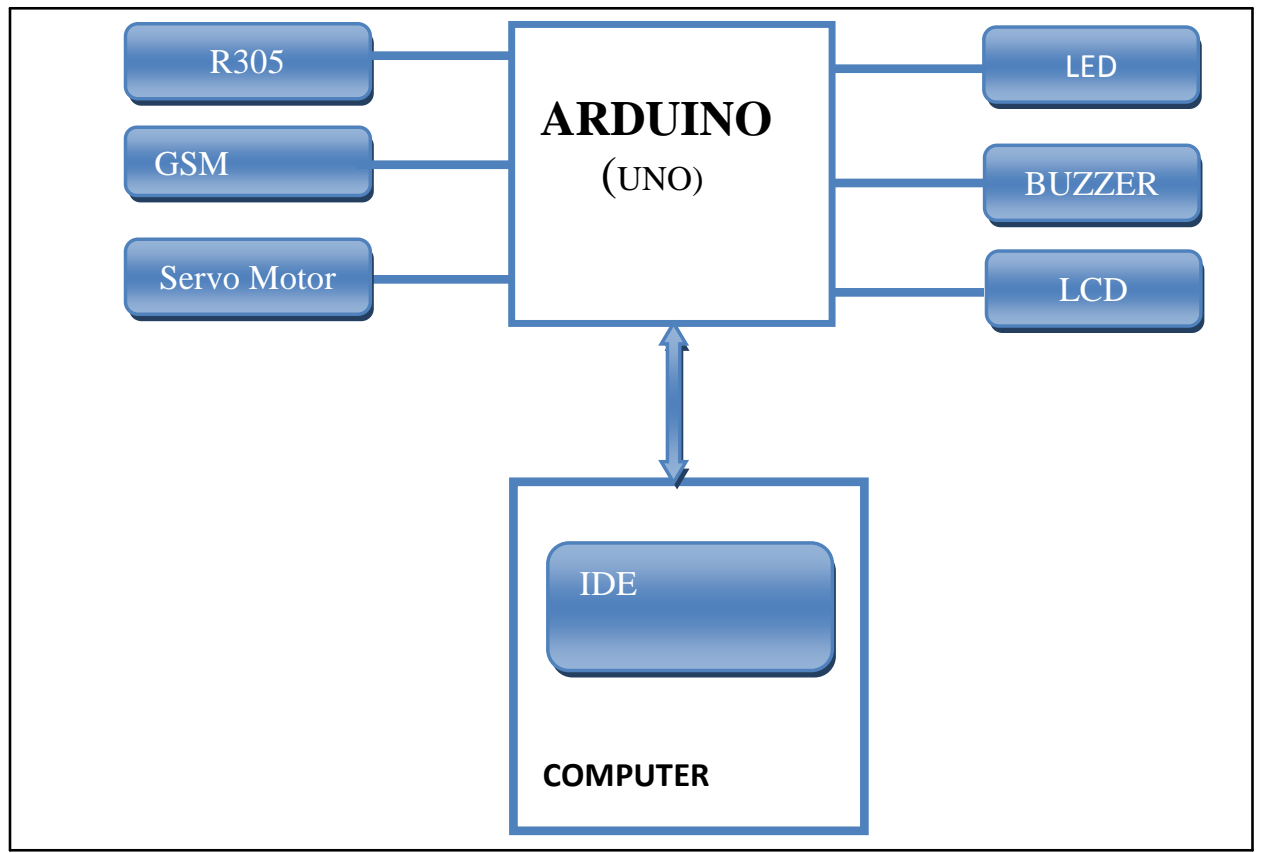

Fig 1.1A block diagram representing the circuit developed

Fingerprint biometric technology provides high level of recognition accuracy. The skin on our palms and soles exhibits a flow like pattern of ridges called friction ridges which makes fingerprint so unique identification for everyone. R305 scans the fingerprints of users and uses for ensuring authentication and follow the necessary steps as shown in Figure 1.2

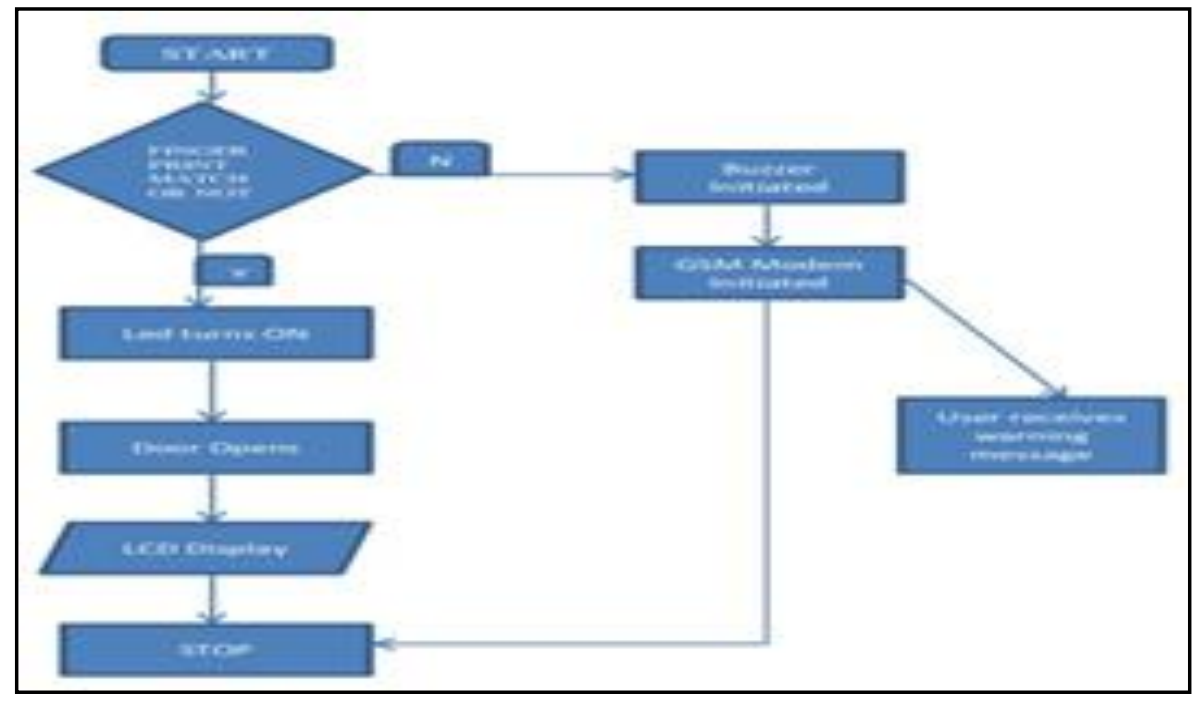

Fig1.2: Working flow chart 


\section{Monitoring Arduino Reading}

Arduino enables users to monitor various Arduino enables users to monitor various kinds of sensors such as finger print sensor and motion detectors in real-time. It has 14 digital input/output pins (of which 6 can be used as PWM outputs), 6 analog inputs, a USB connection, a power jack, a reset button.

The ATmega328 microcontroller embedded on the Arduino board contains the analog-to-digital converter (ADC), which converts the analog input signal to a number between 0 and 1023.The analog and digital pins on the Arduino board can serve as general purpose input and output pins (GPIO).Usually the integer number is always proportional to the amount of the voltage being applied to the analog input. Any sensor operating on 5 volts can be directly connected to the Arduino board. The biometric prototype has been implemented on the board

\section{Controlling Actuators}

The Arduino gateway controls the buzzer and initialization of GSM modem when there is negative match in R305 module. In this system it has two section one the transmitter section where the power supply of $5 \mathrm{v}$ is given through Arduino. The other one is the receiver section where input is given through finger print sensor module and implemented on Arduino. The Arduino receives the digital output and display in the LCD when there is a positive match or else it initiates the buzzer and the GSM modem.In this section we have used LCD to display the result of finger print biometric technology. The system is designed using the Arduino(IDE) platform.

\section{Experimental Evaluation}

In order to implement and demonstrate the system developed theoretically, we created a prototype that represents the system. Thus the whole system that is being developed is given below, (Fig. no: 1.3).

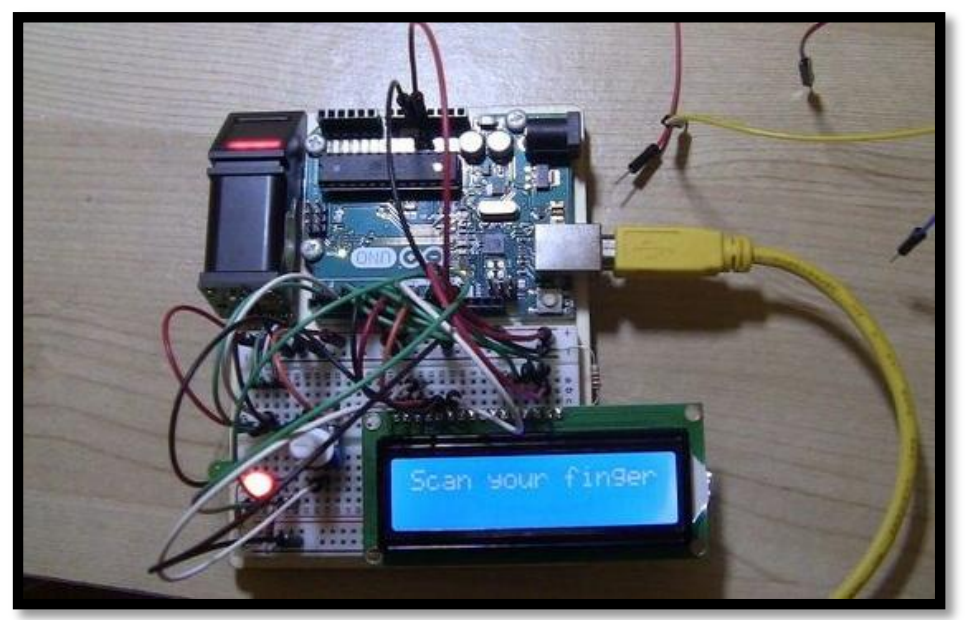

Fig.1.3: Prototype Circuit

\section{Discussions and Conclusions}

The biometric finger print system provides good solution to the security. A novel architecture for an economic Finger print biometric technology is proposed and implemented in this paper. It gives basic idea of how to detect the finger print using R305, Arduino Uno and Ardiono (IDE). The cost of this technology is very economical. This project uses low cost off the shelf components, and is based on Arduino platform which is FOSS (Free Open Source Software). So the overall implementation cost is very cheap and is affordable by a common person. This low cost system is designed to improve the security system. It provides accurate finger recognition results eliminating the error where possible. For future work, some recommendation can be made like, addition of cameras for taking a snapshot for a negative match result. Retina scan security system can also be added for better security purpose. The whole system can be fabricated as economic commercial hardware package.

[1]. https://en.wikipedia.org/wiki/Password

\section{References}

[2]. https://www.techopedia.com/definition/8797/password-protection

[3]. http://www.technovelgy.com/ct/technology-article.asp

[4]. https://en.wikipedia.org/wiki/RFID_skimming

[5]. https://www.arduino.cc/en/Main/ArduinoBoardUno

[6]. https://en.wikipedia.org/wiki/Arduino 
[7]. http://www.sunrom.com/p/finger-print-sensor-r305

[8]. http://www.microcontroller-project.com/16x2-lcd-working.html

[9]. http://www.ijsrp.org/research-paper-0716/ijsrp-p5552.pdf

[10]. http://www.bharathielectronics.in/gsm-module.html

[11]. http://www.rhydolabz.com/documents/gps_gsm/sim900_rs232_gsm_modem_opn.pdf

[12]. https://en.wikipedia.org/wiki/Servomotor 